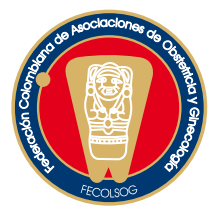

INVESTIGACIÓN ORIGINAL

\title{
BAJO PESO AL NACER: EXPLORACIÓN DE ALGUNOS FACTORES DE RIESGO EN EL HOSPITAL UNIVERSITARIO SAN JOSÉ EN POPAYÁN (COLOMBIA)
}

\section{Low birth weight: exploring of some risk factors at the San José teaching hospital in Popayán, Colombia} Virginia Daza, M.D. *, Wilson Jurado, M.D. *, Diana Duarte, M.D. *, Ignasi Gich, M.D. **, Carlos Hernán Sierra-Torres, M.D.***, Mario Delgado-Noguera, M.D., MSc****

Recibido: marzo 9/09 - Aceptado: junio 8/09

\section{RESUMEN}

Introducción: el bajo peso al nacer (BPN) es un importante problema de salud pública en los países en desarrollo y es un indicador de la salud materno-infantil. El peso al nacer por debajo de 2.500 g contribuye a problemas de salud materno-infantiles de corto y largo plazo.

Objetivo: establecer los factores asociados con el bajo peso al nacer en el Hospital Universitario San José, Popayán (Colombia), en el período comprendido entre los años 2005 y 2006.

Métodos y materiales: estudio de casos y controles. Los casos $(n=344)$ fueron los recién nacidos con peso menor a $2.500 \mathrm{~g}$ que nacieron en este hospital. Los controles $(n=483)$ fueron recién nacidos con peso mayor a $2.500 \mathrm{~g}$ que nacieron el mismo día. Se realizó una entrevista en mujeres después del parto para recoger información acerca de los

* Médicos Pediatras egresados de la Universidad del Cauca. Popayán (Colombia).

** Servicio de Epidemiología y Salud Pública del Hospital de la Santa Creu i Sant Pau. Barcelona (España).

*** Profesor asociado, departamento de Ciencias Fisiológicas, Facultad de Ciencias de la Salud, Unidad de Epidemiología Clínica, Universidad del Cauca. Popayán (Colombia).

***** Profesor asociado, departamento de Pediatría, Facultad de Ciencias de la Salud, Unidad de Epidemiología Clínica, Universidad del Cauca. Correspondencia: Centro Cochrane Iberoamericano, St. Antoni M. Claret, 171, 08041 Barcelona (España). Correo electrónico: mariodelg@gmail.com factores de riesgo; y se estimó el Odds Ratio (OR, por sus siglas en inglés) y sus respectivos intervalos de confianza (IC95\%) utilizando un modelo de regresión logística.

Resultados: como factores de protección se consideraron: antecedentes de estrato socioeconómico medio/superior $(\mathrm{OR}=0,33$; IC95\% 0,12-0,91), sexo femenino del recién nacido $(\mathrm{OR}=0,73$; IC95\% 0,55-0,98), tener más de 5 controles prenatales $(\mathrm{OR}=0,54$; IC95\% 0,39-0,75) y no tener antecedentes previos de BPN (OR=0,30; IC95\% 0,15-0,58). Entre los factores de riesgo se encontraron: antecedentes de infecciones del tracto urinario $(\mathrm{OR}=1,78$; IC95\% 1,30-2,45) y cesárea anterior $(\mathrm{OR}=1,88$; IC95\% 1,13-3,16).

Conclusión: el bajo peso al nacer está asociado con el estrato socioeconómico, por lo que intervenir en la falta de equidad social es un factor de importancia para la reducción del BPN en el área de influencia de este centro de atención terciario.

Palabras clave: factores de riesgo, estudios de casos y controles, recién nacido de bajo peso, mortalidad infantil, Colombia.

\section{SUMMARY}

Introduction: low birth weight (LBW) is a major public health problem and maternal-infant health 
indicator in developing countries. A birth weight below $2.500 \mathrm{~g}$ contributes towards poor short- and long-term maternal-infant health outcomes.

Objective: establishing risk factors for LBW in the San José teaching hospital in Popayán, Colombia, 2005-2006.

Methods and materials: this was a case-control study. Cases $(n=344)$ were newborn $(<2.500 \mathrm{~g})$ who had been born in this hospital; the controls $(n=483)$ were newborn $(>2.500 \mathrm{~g})$ who were born on the same day. An interview was conducted to collect information regarding risk factors from post-partum females. A logistic regression model was used for estimating risk factors by Odds Ratio (OR) with confidence intervals (95\%CI).

Results: having a middle/upper socio-economic class background ( $\mathrm{OR}=0,33$; 95\% CI 0,12-0,91), the newborn being female $(\mathrm{OR}=0,73 ; 95 \% \mathrm{CI}$ $0,55-0,98)$, having had more than 5 prenatal controls (OR $=0,54$; 95\%CI 0,39-0,75), having no background of previous LBW $(\mathrm{OR}=0,30$; 95\%CI 0,15-0,58) were protective factors for LBW. Having a background of urinary tract infection $(\mathrm{OR}=1,78$; 95\% CI 1,30-2,45) and having undergone a previous caesarean section $(\mathrm{OR}=1,88 ; 95 \% \mathrm{CI} 1,13-3,16)$ were low birth weight risk factors.

Conclusion: LBW was thus seen to be associated with coming from a lower socio-economic class. Interventions regarding the lack of social equity is therefore an important factor in reducing LBW in this third-level hospital's area of influence.

Key words: risk factor, case-control study, infant, low birth weight, infant mortality, Colombia.

\section{INTRODUCCIÓN}

La definición de bajo peso al nacer (BPN) es empleada por la Organización Mundial de la Salud (OMS) para referirse a los niños con peso inferior a $2.500 \mathrm{~g}$ al momento de nacer. ${ }^{1}$ El BPN evidencia la salud materno-fetal durante la gestación, y es un factor directamente relacionado con la morbilidad y la mortalidad neonatales e infantiles y con el bienestar de la madre y su hijo en la etapa posparto, donde tiene un reconocido impacto. ${ }^{2,3} \mathrm{~A}$ nivel global, las muertes neonatales corresponden a 37\% de la mortalidad en menores de 5 años y el BPN es la segunda causa de muerte después de la prematuridad en recién nacidos. ${ }^{4,5}$ De modo que los niños con BPN tienen 5 a 30 veces más riesgo de morir que los nacidos con peso normal y éste está igualmente asociado con mayor probabilidad de infección, desnutrición, parálisis infantil, deficiencias mentales y trastornos del aprendizaje.$^{6-8}$ Asimismo, el BPN se ha asociado, en la etapa adulta, con una mayor predisposición a diabetes y enfermedades cardiovasculares. ${ }^{9}$

La OMS ha estimado en Latinoamérica una prevalencia de 9,26\% de BPN con fluctuaciones regionales y dentro de los países del área. ${ }^{6}$ En Colombia, la prevalencia de BPN es de $9 \% ;^{10}$ y con un sistema de salud en crisis, a los hospitales públicos de tercer nivel con problemas finacieros se remiten, para la atención del parto y desde niveles primarios, las madres con embarazos de alto riesgo. Igualmente, en estos hospitales se atienden partos de embarazos sin complicaciones. ${ }^{11}$

Los problemas de la atención en los primeros niveles y las dificultades del acceso de la población a los servicios se manifiestan en la atención especializada del tercer nivel, como en el caso del BPN. Por esta razón, es de relevancia estudiar aquellos factores de riesgo que afectan a las madres con hijos con posible BPN en los hospitales de tercer nivel. Esta perspectiva permitirá al personal médico, a las autoridades en salud y a los responsables de la política pública, observar algunos aspectos del BPN que podrían ser objeto de intervenciones preventivas que se sitúan por fuera de la atención de ese nivel y así evitar sus consecuencias y la saturación de los servicios.

La literatura médica ha reconocido condiciones socioeconómicas, étnicas, maternas, fetales y medioambientales relacionadas con el BPN. ${ }^{12,13}$ A su vez, estas condiciones presentan diferencias regionales en cuanto a su distribución y relevancia. Por ejemplo, en la región europea, el nacimiento 
prematuro es el factor que más se relaciona con el BPN mientras que en países en vías de desarrollo lo es el Retraso del Crecimiento Intrauterino (RCIU). ${ }^{14}$ En Canadá, se ha observado que el ingreso económico de las familias está asociado con el parto pretérmino y con niños con bajo peso para la edad gestacional. ${ }^{15}$ Además, en publicaciones de Latinoamérica se ha establecido que el bajo nivel socioeconómico es uno de los factores independientes más relevantes relacionados con el BPN, ${ }^{12,16}$ de tal manera que la inequidad social toma un papel importante en este problema materno-infantil y en sus consecuencias deletéreas tempranas y tardías.

En este estudio, postulamos como hipótesis a priori que en el área de estudio (un hospital de tercer nivel que presta atención a la región del Cauca, en el suroccidente de Colombia), el bajo estrato socioeconómico constituye un factor de riesgo para el BPN.

\section{MÉTODOS}

Se realizó un estudio de casos y controles entre el 15 de noviembre de 2005 y el 15 de noviembre de 2006 en el Hospital Universitario San José de tercer nivel de atención, en Popayán, ciudad intermedia del suroccidente colombiano. Este hospital presta sus servicios a pacientes del régimen subsidiado (sistema de aseguramiento público, subsidiado por el Estado) y contributivo (sistema de aseguramiento privado). Para el tamaño muestral, teniendo en cuenta que el lugar del estudio es un hospital público que atiende principalmente a población pobre, se estimó una frecuencia de estrato socioeconómico bajo de 40\% para los casos y de 30\% para los controles. El BPN se definió como aquel recién nacido $(\mathrm{RN})$ que hubiera pesado menos de $2.500 \mathrm{~g}$. En el grupo de casos, se tomaron los RN con BPN nacidos en ese período ( $n=344)$; y en el grupo de controles, a los RN que pesaron más de $2.500 \mathrm{~g}(\mathrm{n}=483)$, nacidos el mismo día en el mismo hospital. No se hizo muestreo aleatorio de los controles; y tanto casos como controles procedían del área de influencia del hospital San José.
Para la clasificación de peso en la edad gestacional, el cual indica el grado de riesgo al momento del nacimiento, se empleó la gráfica de Lubchenco, que clasifica al recién nacido en PEG (Pequeños para la Edad Gestacional) AEG (Adecuados para la Edad Gestacional) y GEG (Grandes para la Edad Gestacional). ${ }^{17}$

Como factores de exclusión, se consideró que la madre no firmara el consentimiento informado y se descartaron los controles que presentaban dificultades para obtener todos los datos de las madres que egresaban durante los fines de semana. Respecto a la recolección de la información, se diseñó un instrumento que se basó principalmente en el formulario desarrollado por el Centro Latino Americano de Perinatología (CLAP) para la vigilancia epidemiológica de la salud materna y perinatal de Latinoamérica desde $1983 .{ }^{18}$ Sus dominios estuvieron conformados por antecedentes sociodemográficos, antecedentes clínicos, riesgo clínico del embarazo, antecedentes ambientales, conductas de riesgo y características de los RN, que en total, reunían información de 38 variables. El registro de la información se realizó a partir de la historia clínica materna y de la entrevista que los investigadores (Daza V., Duarte D., Jurado W.) efectuaban a cada una de las madres durante el período de puerperio hospitalario.

Después de obtener los datos proporcionados por los instrumentos, éstos fueron analizados en el paquete estadístico SPSS 14.0. Una vez verificados, se describieron las variables categóricas por medio de proporciones, y las continuas con medidas de tendencia central y de dispersión.

Igualmente, se llevó a cabo el análisis univariado con los métodos estadísticos Chi2 y T-test para observar la posible asociación de las diferentes variables categóricas o continuas, respectivamente, y la variable de respuesta, BPN; y se calcularon los Odds Ratios (OR) con su correspondiente IC95\%. El grupo de referencia fue el considerado como de riesgo para cada variable, i.e. para el régimen de salud colombiano este grupo lo conformaron las madres que pertenecían a la categoría de subsidiado. 
Con las variables que resultaron ser significativas a un nivel menor de 0,05 en el análisis bivariado, se llevó a cabo el análisis multivariado por medio de regresión logística. Dicha decisión se tomó para limitar el número de variables incluidas finalmente en la aproximación multivariante, y así evitar en lo posible una sobredimensión del error tipo 1. Se tomaron en cuenta tres modelos: el modelo 1, con las variables sociodemográficas; el modelo 2, con los antecedentes reproductivos y el modelo 3, con los antecedentes de morbilidad y de exposición durante el embarazo. La variable ajustada que resultaba significativa en el primer modelo se añadía a las variables del segundo para el análisis con la regresión logística y las del segundo para el análisis del tercero. Este método de análisis multivariado ha sido previamente descrito. ${ }^{12}$

\section{RESULTADOS}

De un total de 1.095 nacimientos ocurridos en el Hospital Universitario San José de tercer nivel entre diciembre de 2005 a diciembre de 2006, se incluyeron en el estudio 827 recién nacidos representando 75,5\% de los casos. La media de peso al nacer fue de 2.639 g con una Desviación Estándar (DS) de $727 \mathrm{~g}$ mientras que la mediana fue de 2.700 g. De ellos, 19,1\% fueron clasificados como PEG, $74,1 \%$ como AEG y 6,7\% como GEG. En total, 344 fueron los RN con BPN en ese período de tiempo (i.e. todos los casos) de tal manera que la prevalencia de BPN en este centro de tercer nivel sobre el total de los nacimientos en ese período fue de 31,4\% (344/1.095).

Las características sociodemográficas de las madres se presentan en la tabla 1. En conclusión, se observaron diferencias significativas en la edad materna entre los casos y los controles mientras que no se encontraron diferencias significativas en las restantes variables sociodemográficas.

En cuanto a los antecedentes reproductivos, se advirtieron diferencias significativas entre los casos y los controles en el número mayor o menor a cinco controles prenatales, en la paridad, en las cesáreas previas y en madres con antecedentes de hijos con BPN (tabla 2).

Los antecedentes de morbilidad y de exposición durante el embarazo se muestran en la tabla 3. Se encontraron diferencias significativas en embarazadas con antecedentes de Infección de Vías Urinarias (IVU), aquellas que consumieron suplementos de hierro y ácido fólico durante el embarazo y las que estuvieron expuestas a humo de leña.

De otra parte, se llevaron a cabo los tres modelos planeados, como puede observarse en la tabla 4. En los tres se observó que el estrato socioeconómico medio/alto es un factor protector significativo para el BPN y es el factor que permanece en todos los modelos. En el modelo 3 se encontró que los factores protectores presentes en el último modelo fueron el sexo femenino del RN (OR=0,73; IC95\% 0,55$0,98)$, haber asistido a más de 5 controles prenatales $(\mathrm{OR}=0,54$; IC95\% 0,39-0,75) y no haber tenido hijos con BPN (OR=0,30; IC95\% 0,15-0,58). Fueron factores de riesgo, tener antecedentes de cesáreas $(\mathrm{OR}=2,36$; IC95\% 0,72-3,25) y de infección de vías urinarias durante el embarazo $(\mathrm{OR}=1,79$; IC95\% 1,30-2,45) (Modelo 3).

\section{DISCUSIÓN}

El BPN continúa siendo un factor primordial en la mayoría de las muertes infantiles y en la morbilidad infantil a largo plazo. La reducción de la incidencia del BPN está dentro de una de las principales metas en el propósito de reducir la mortalidad infantil de los “Objetivos del Milenio" según la declaración de las Naciones Unidas en 2002, y por lo tanto, es un indicador del monitoreo del alcance de dichas metas en el 2015. ${ }^{19}$ En el Hospital Universitario San José de Popayán, se llevó a cabo una investigación previa que demostró que el BPN fue un factor de riesgo importante para la mortalidad neonatal $\left(\mathrm{OR}=2,59\right.$; IC95\% 1,01-6,60). ${ }^{20}$ Igualmente, se concluyó que el reconocimiento de las barreras socioeconómicas, culturales y logísticas para la búsqueda de cuidado y el acceso de las madres a los servicios de salud tiene posibilidades de disminuir su frecuencia y sus consecuencias. ${ }^{3}$ 
Tabla 1. Características sociodemográficas de las madres de recién nacidos con peso adecuado y bajo peso al nacer. Hospital Universitario San José, Popayán (Colombia) 2005-2006.

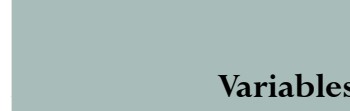

Total

Edad*

Media \pm DS

$\leq 20$

$20-30$

$>30$

\section{Procedencia}

Rural

Urbano

\section{Desplazada}

No

Sí

\section{Estado civil}

Casada

No casada

\section{Educación}

Ninguna

Primaria

Secundaria

Universitaria

\section{Estrato socioeconómico}

Bajo

Medio/alto

\section{Régimen de salud}

Vinculado

Subsidiado

Contributivo

$* p \leq 0,05$

\section{Peso al nacer}

n (\%)

\begin{tabular}{c|c}
$<2.500 \mathrm{~g}$ & $>2.500 \mathrm{~g}$ \\
\hline 344 & 483
\end{tabular}

\begin{tabular}{c|c|c}
\hline $24,6 \pm 7,4$ & $25,7 \pm 8,1$ & \\
\hline $125(36,4)$ & $152(31,4)$ & 1,0 \\
\hline $136(39,5)$ & $196(40,6)$ & $0,84(0,61-1,16)$ \\
\hline $83(24,1)$ & $135(28,0)$ & $0,75(0,52-1,07)$
\end{tabular}

$139(40,4)$

$205(59,6)$
$200(41,4)$

$283(58,6)$

$469(97,1)$

$14(2,9)$

$9(2,6)$

$264(76,7)$

$80(23,3)$

$371(76,8)$

$112(23,2)$

$1,00(0,72-1,39)$
OR (IC 95\%) 
Tabla 2. Antecedentes reproductivos de las madres de recién nacidos con peso adecuado y bajo peso al nacer. Hospital Universitario San José, Popayán (Colombia) 2005-2006.

\begin{tabular}{|c|c|c|c|}
\hline \multirow[t]{2}{*}{ Variables } & \multicolumn{2}{|c|}{$\begin{array}{c}\text { Peso al nacer } \\
\text { n (\%) }\end{array}$} & \multirow[t]{2}{*}{ OR (IC 95\%) } \\
\hline & $<2.500 \mathrm{~g}$ & $>2.500 \mathrm{~g}$ & \\
\hline Total & 344 & 483 & \\
\hline \multicolumn{4}{|l|}{ Sexo del RN } \\
\hline Masculino & $169(49,1)$ & $208(43,1)$ & 1,0 \\
\hline Femenino & $175(50,9)$ & $275(56,9)$ & $0,78(0,59-1,03)$ \\
\hline \multicolumn{4}{|l|}{ Control prenatal* } \\
\hline Media \pm DS & $4,20 \pm 2,57$ & $5,15 \pm 2,61$ & \\
\hline$\leq 5$ & $225(65,4)$ & $250(51,8)$ & 1,0 \\
\hline$>5$ & $119(34,6)$ & $233(48,2)$ & $0,57(0,43-0,76)$ \\
\hline \multicolumn{4}{|l|}{ Talla materna en $\mathrm{cm}$} \\
\hline Media $\pm \mathrm{DS}$ & $155,2 \pm 6,86$ & $155,5 \pm 6,80$ & \\
\hline$\leq 149$ & $56(16,3)$ & $88(18,2)$ & 1,0 \\
\hline$>149$ & $288(83,7)$ & $395(81,8)$ & $1,14(0,79-1,65)$ \\
\hline \multicolumn{4}{|l|}{ Paridad* } \\
\hline Primípara & $132(38,4)$ & $118(24,4)$ & 1,0 \\
\hline Multípara & $212(61,6)$ & $365(75,6)$ & $0,52(0,38-0,70)$ \\
\hline \multicolumn{4}{|l|}{ Período intergenésico } \\
\hline$>12$ & $158(46,5)$ & $242(50,2)$ & 1,0 \\
\hline$\leq 12$ & $182(53,5)$ & $240(49,8)$ & $1,16(0,88-1,53)$ \\
\hline \multicolumn{4}{|l|}{ Cesáreas* } \\
\hline Ninguna & $125(36,3)$ & $257(53,2)$ & 1,0 \\
\hline Una cesárea & $182(52,9)$ & $176(36,4)$ & $2,13(1,58-2,86)$ \\
\hline Más de una cesárea & $37(10,8)$ & $50(10,4)$ & $1,52(0,94-2,45)$ \\
\hline \multicolumn{4}{|l|}{ Antecedentes de aborto } \\
\hline Sí & $42(12,2)$ & $80(16,6)$ & 1,0 \\
\hline No & $302(87,8)$ & $403(83,4)$ & $0,70(0,47-1,05)$ \\
\hline \multicolumn{4}{|l|}{ Antecedentes de BPN* } \\
\hline Sí & $33(96,7)$ & $17(3,5)$ & 1,0 \\
\hline No & $311(90,4)$ & $466(96,5)$ & $0,34(0,19-0,63)$ \\
\hline
\end{tabular}


Tabla 3. Antecedentes de morbilidad y de exposición durante el embarazo de las madres de recién nacidos con peso adecuado y bajo peso al nacer. Hospital Universitario San José, Popayán (Colombia) 2005-2006.

\begin{tabular}{|c|c|c|c|}
\hline \multirow{2}{*}{ Variables } & \multicolumn{2}{|c|}{$\begin{array}{c}\text { Peso al nacer } \\
\text { n (\%) }\end{array}$} & \multirow{2}{*}{ OR (IC 95\%) } \\
\hline & $<2.500 \mathrm{~g}$ & $>2.500 \mathrm{~g}$ & \\
\hline Total & 344 & 483 & \\
\hline
\end{tabular}

\section{Hipertensión}

\begin{tabular}{|c|c|c|c|}
\hline Sí & $332(96,5)$ & $467(96,7)$ & 1,0 \\
\hline No & $12(3,5)$ & $16(3,3)$ & $1,05(0,49-2,26)$ \\
\hline \multicolumn{4}{|c|}{ Infección de Vías Urinarias (IVU)* } \\
\hline No & $206(59,9)$ & $350(72,5)$ & 1,0 \\
\hline Sí & $138(40,1)$ & $133(27,5)$ & $1,76(1,31-2,36)$ \\
\hline \multicolumn{4}{|c|}{ Suplementación con Hierro* } \\
\hline No & $277(80,5)$ & $422(87,4)$ & 1,0 \\
\hline Sí & $67(19,5)$ & $61(12,6)$ & $0,60(0,41-0,87)$ \\
\hline \multicolumn{4}{|c|}{ Suplementación con ácido fólico* } \\
\hline No & $276(80,2)$ & $423(87,6)$ & 1,0 \\
\hline Sí & $68(19,8)$ & $60(12,4)$ & $0,58(0,39-0,84)$ \\
\hline \multicolumn{4}{|c|}{ Alcohol } \\
\hline No & $333(96,8)$ & $476(98,6)$ & 1,0 \\
\hline Sí & $11(3,2)$ & $7(1,4)$ & $2,25(0,86-5,85)$ \\
\hline \multicolumn{4}{|c|}{ Café } \\
\hline No & $51(14,8)$ & $64(13,3)$ & 1,0 \\
\hline Sí & $293(85,2)$ & $419(86,7)$ & $0,88(0,59-1,30)$ \\
\hline \multicolumn{4}{|c|}{ Tabaco } \\
\hline No & $338(98,3)$ & $476(98,6)$ & 1,0 \\
\hline Sí & $6(1,7)$ & $7(1,4)$ & $1,21(0,40-3,62)$ \\
\hline \multicolumn{4}{|c|}{ Plaguicidas } \\
\hline No & $320(93,0)$ & $462(95,7)$ & 1,0 \\
\hline Sí & $24(7,0)$ & $21(4,3)$ & $1,65(0,90-3,01)$ \\
\hline \multicolumn{4}{|c|}{ Humo de leña } \\
\hline No & $144(41,9)$ & $235(48,7)$ & 1,0 \\
\hline Sí & $200(58,1)$ & $248(51,3)$ & $1,32(1,00-1,74)$ \\
\hline
\end{tabular}

$* p \leq 0,05$ 
más elevados que las ciudades mencionadas, el índice de necesidades básicas insatisfechas es mayor que el del promedio de Colombia ${ }^{24}$ y sus servicios de salud han tenido frecuentes altibajos después de la promulgación de la ley 100, la ley que reglamenta el sistema de salud colombiano. Por lo tanto, el Hospital Universitario San José, de carácter público, al igual que otros hospitales universitarios del país, dejan de ser fundamentales como instituciones de salud integral y colectiva bajo esa ley y bajo el modelo económico neoliberal que domina el Estado Colombiano. ${ }^{25}$

De otra parte, las mujeres embarazadas con un inadecuado control prenatal tienen mayor riesgo de morbilidad perinatal. ${ }^{26}$ En el estudio, tener un número de controles prenatales mayor a cinco fue un factor protector; este hecho también se había presentado en el estudio realizado en Medellín. ${ }^{22} \mathrm{El}$ promedio de visitas de control prenatal fue menor en los casos que en los controles, lo cual indica que las autoridades de salud deben promover un control eficiente que sea precoz, periódico, integral y de amplia cobertura. ${ }^{23}$

Las madres que han tenido hijos con BPN, tienen una mayor posibilidad de que al tener nuevamente hijos, éstos nazcan con BPN. La presente investigación corroboró este hecho encontrado en la literatura médica. Por consiguiente, investigar exhaustivamente este dato en la historia clínica del control prenatal, permitiría intervenir durante la gestación modificando factores asociados al BPN como la mala nutrición o la anemia. ${ }^{23}$ La suplementación con hierro y ácido fólico mostraron su efecto protector en el análisis bivariado si bien no persistieron como protectores en el modelo ajustado final. De modo que la suplementación con hierro durante el embarazo es efectiva para reducir el BPN y la mortalidad perinatal y neonatal. ${ }^{27}$

Asimismo, un factor modificable en el cuidado individual de la embarazada con riesgo de BPN es el diagnóstico y tratamiento oportuno de la IVU. ${ }^{23}$ En el estudio, esta condición se mostró claramente como un factor de riesgo.
Haber tenido una o más cesáreas previas también se mostró como un factor de riesgo para el BPN; un factor fácilmente detectable en el control prenatal que permite vigilar a la embarazada con este antecedente de forma más cercana y persistente.

Por otro lado, este estudio de casos y controles tiene como debilidad principal la elección no aleatoria de los controles. Sin embargo, tiene la fortaleza de basarse en un tamaño muestral adecuado para detectar diferencias en el estrato socioeconómico, factor no tan cercano al evento resultado, y de tomar como casos todos los RN de bajo peso durante el período de un año. La alta prevalencia encontrada del BPN se debe a que el lugar del estudio corresponde a un centro de referencia regional de madres cuyos embarazos son de riesgo.

En conclusión, la investigación demostró que en la población estudiada hay factores modificables desde el ámbito clínico como la pesquisa, el tratamiento y el seguimiento de la IVU. Factores de los servicios de salud como la promoción de un adecuado control prenatal, y principalmente, la ampliación de la disponibilidad y la calidad de la atención en el ámbito prenatal y la elevación del nivel de vida de las mujeres y sus familias, mejorarían varios problemas de la salud materno-infantil, entre ellos el BPN. ${ }^{28}$ Esto se facilitaría con una instauración de una política pública de salud en Colombia que enfrente las desigualdades sociales, una idea que no es nueva pues desde mediados del siglo XIX, el fundador de la teoría de la patología celular, Rudolf Virchow, afirmaba que esas desigualdades son la raíz causal de muchas enfermedades y condiciones,${ }^{29}$ entre ellas el problema del BPN, cuyo ámbito de atención no se puede centrar únicamente en la clínica.

\section{REFERENCIAS}

1. Organización Panamericana de la Salud. Condiciones de Salud del Niño en las Américas. Publicación Científica No. 381. Washington, D.C.; 1979.

2. Valero De Bernabé J, Soriano T, Albaladejo R, Juarranz M, Calle ME, Martínez D, et al. Risk factors for low birth weight: a review. Eur J Obstet Gynecol Reprod Biol 2004;116:3-15. 
3. Moss W, Darmstadt GL, Marsh DR, Black RE, Santosham M. Research priorities for the reduction of perinatal and neonatal morbidity and mortality in developing country communities. J Perinatol 2002;22:484-95.

4. WHO. Causes of death in neonates and children under five in the African Region (2004). WHO. The Global Burden of disease: 2004 update (2008).

5. Thornton JG. Perinatal mortality rises both with prematurity and with the degree to which the baby's birthweight is below that expected for gestational age. Eur J Obstet Gynecol Reprod Biol 2001;95:5

6. WHO. Low birthweight. Country, regional and global estimates 2005. Visitado en 2009 May 29. Disponible en: http://www.who.int/reproductivehealth/publications/low_birthweight/index

7. Jones G, Steketee RW, Black RE, BhutaZA, Morris S; Bellagio Child Survival Study Group. How many child deaths can we prevent this year? Lancet 2003;362:65-71.

8. Vincer MJ, Allen AC, Joseph KS, Stinson DA, Scott $\mathrm{H}$, Wood E. Increasing prevalence of cerebral palsy among very preterm infants: a population-based study. Pediatrics 2006;118:e1621-6.

9. Barker DJP, Forsén T, Uutela A, Osmond C, Eriksson JG. Size at birth and resilience to effects of poor living conditions in adult life: longitudinal study. BMJ 2001;323:1273-6.

10. WHO. Risk factors 2009. Visitado en 2009 May 29. Disponible en: http://www.who.int/whosis/whostat/ EN_WHS09_Table5.pdf

11. Paredes N. La salud pública: entierro definitivo. En: Borrero García C. (Ed). Reelección: el embrujo continúa, segundo año de gobierno de Álvaro Uribe Vélez. Bogotá: Plataforma Colombiana de Derechos Humanos, Democracia y Desarrollo; 2004. p. 111.

12. Torres-Arreola LP, Constantino-Casas P, FloresHernández S, Villa-Barragán JP, Rendón-Macías E. Socioeconomic factors and low birth weight in Mexico. BMC Public Health 2005;5:20.

13. Demissie K, Rhoads GG, Ananth CV, Alexander GR, Kramer MS, Kogan MD, et al. Trends in preterm birth and neonatal mortality among blacks and whites in the United States from 1989 to 1997. Am J Epidemiol 2001;154:307-15.

14. Villar J, Belizán JM. The relative contribution of prematurity and fetal growth retardation to low birth weight in developing and developed societies. Am J Obstet Gynecol 1982;143:793-8.
15. Joseph KS, Liston RM, Dodds L, Dahlgren L, Allen AC. Socioeconomic status and perinatal outcomes in a setting with universal access to essential health care services. CMAJ 2007;177:583-90.

16. Mejía Salas H, Córdova G, Fernández JC. Factores de riesgo para muerte perinatal en el hospital Los Andes, El Alto, Bolivia. Cuad Hosp Clin 2000;47:47-62.

17. Lubchenco LO, Searls DT, Brazie JV. Neonatal mortality rate: relationship to birth weight and gestational age. J Pediatr 1972;81:814-22.

18. Centro Latinoamericano de Perinatología, Salud de la Mujer y Medicina Reproductiva (CLAP). Visitado en 2009 Ene 23. Disponible en: http://www.clap.opsoms.org

19. ONU. Objetivo: reducir la mortalidad de los niños menores de 5 años. Visitado en 2009 Jun 2. Disponible en: http://www.un.org/spanish/millenniumgoals/ childhealth.shtml.

20. Delgado M, Muñoz A, Orejuela L, Sierra CH. Algunos factores de riesgo para mortalidad neonatal en un hospital de III nivel, Popayán. Colomb Med 2003;34:179-85.

21. Isaza S, Morales Y. Retardo del crecimiento intrauterino. En: Ucrós S Caicedo A, Llano G. Guías de pediatría basadas en la evidencia. Bogotá: Ed. Panamericana; 2003.

22. Vélez-Gómez MP, Barros FC, Echavarría-Restrepo LG, Hormaza-Angel MP. Prevalencia de bajo peso al nacer y factores maternos asociados: Unidad de Atención y Protección Materno Infantil de la Clínica Universitaria Bolivariana, Medellín, Colombia. Rev Colomb Obstet Ginecol 2006;57:264-70.

23. Ortiz EI. Estrategias para la prevención del bajo peso al nacer en una población de alto riesgo, según la medicina basada en la evidencia. Colomb Med 2001;32:159-62.

24. Portela H. Perspectiva política de la salud. En: Barona G, Gnecco C, eds. Territorios posibles. Historia, Geografía y Cultura del Cauca. Popayán: Editorial Universidad del Cauca; 2001

25. Abadía CE, Navarrete HG, Martínez AG, Pinilla MY. La crisis de la salud pública en el Instituto MaternoInfantil de Bogotá, Antípoda: Revista de Antropología y Arqueología 2006;(3):199-226.

26. Panamerican Health Organization, PAHO - World Health Organization, WHO. Manual de atención integrada a las enfermedades prevalentes de la infancia. Washington: PAHO-WHO; 2004. 
27. Preziosi P, Prual A, Galan P, Dauda H, Boureima H, Herchberg S. Effect of iron supplementation on the iron status of pregnant women: consequences for newborns. Am J Clin Nutr 1997;66:1178-82.

28. Bhutta ZA, Darmstatd GL, Ransom EI. Utilización de la evidencia investigativa para salvarle la vida a los recién nacidos. Perspectivas de las políticas sobre la salud neonatal. Washington: Save the Children-Population Reference Bureau; 2003.

29. Mackenbach JP. Politics is nothing but medicine at a larger scale: reflections on public health's biggest idea. J Epidemiol Community Health 2009;63:181-4. 